\title{
Targeting Chemokine Receptor CCR4 in Adult T-Cell Leukemia-Lymphoma and Other T-Cell Lymphomas
}

\author{
Kensei Tobinai • Takeshi Takahashi • Shiro Akinaga
}

Published online: 27 April 2012

(C) The Author(s) 2012. This article is published with open access at Springerlink.com

\begin{abstract}
Peripheral T-cell lymphoma (PTCL) is a group of lymphoid malignancy that remains difficult to treat, as most PTCL becomes refractory or relapses, and thus there is an unmet medical need for novel treatment modalities. CC chemokine receptor 4 (CCR4) is expressed in various types of PTCL including adult T-cell leukemia-lymphoma (ATL), which has the worst prognosis among them. A phase II study of a defucosylated, humanized anti-CCR4 monoclonal antibody, mogamulizumab (KW-0761), yielded an overall response rate of $50 \%(13 / 26)$ and a median progression-free survival of 5.2 months in relapsed patients with CCR4positive ATL who had been previously treated with chemotherapy. Mogamulizumab also showed potential efficacy for cutaneous T-cell lymphoma in a US phase I/II study. Further preclinical and clinical investigations are needed to examine whether concomitant use of this novel agent with other agents with different mechanisms of action would be more effective for ATL and other PTCLs.
\end{abstract}

\footnotetext{
K. Tobinai $(\bowtie)$

Department of Hematology, and Hematopoietic Stem Cell

Transplantation, National Cancer Center Hospital,

5-1-1 Tsukiji, Chuo-ku,

Tokyo 104-0045, Japan

e-mail: ktobinai@ncc.go.jp

T. Takahashi

Clinical Development Department, Kyowa Hakko Kirin Co., Ltd,

1-6-1 Ohtemachi, Chiyoda-ku,

Tokyo 100-8185, Japan

e-mail: takeshi.takahashi@kyowa-kirin.co.jp

S. Akinaga

Development Division, Kyowa Hakko Kirin Co., Ltd,

1-6-1 Ohtemachi, Chiyoda-ku,

Tokyo 100-8185, Japan

e-mail: shiro.akinaga@kyowa-kirin.co.jp
}

Keywords Chemokine receptor · CCR4 - Adult T-cell leukemia-lymphoma $\cdot$ ATL $\cdot$ Peripheral T-cell lymphoma . PTCL $\cdot$ Monoclonal antibody $\cdot$ Mogamulizumab $\cdot$ KW-0761

\section{Introduction}

Peripheral T-cell lymphoma (PTCL) represents a small, heterogeneous group of non-Hodgkin lymphoma (NHL) which is derived from more mature T-cells and natural killer (NK) cells, and accounts for approximately $10 \%$ of NHL cases in Western countries [1,2] and for approximately $20 \%-25 \%$ of those in Japan [3, 4]. PTCL, a collective entity of nearly 20 different subtypes defined according to morphology, immunophenotype, genotype, and clinical features [5], can be largely classified into the following two groups according to clinical features including the sites of lesions: (1) cutaneous T-cell lymphoma (CTCL), which is the general term for diseases that initially or mainly occur in the skin, and (2) PTCL other than CTCL. Treatment strategies have been separately developed for these two groups [6].

Treatment options are substantially different for B-cell and T-cell lymphomas. Rituximab, an anti-CD20 monoclonal antibody, was developed for the treatment of B-cell lymphomas. The introduction of this agent into clinical practice has greatly improved the prognosis of patients with B-cell lymphoma [7]. Recently, bendamustine, which has little cross resistance with other chemotherapeutic agents presumably associated with its unique chemical structure of an alkylating agent and a nucleoside analog, has been developed as effective treatment of relapsed or refractory B-cell lymphoma, considering its lack of cross resistance with other chemotherapeutic agents [8]. However, PTCL remains extremely difficult to treat, because most PTCL subtypes become refractory to even aggressive chemotherapy 
regimens or relapse, with the exception of anaplastic lymphoma kinase-positive anaplastic large cell lymphoma $\left(\mathrm{ALK}^{+}\right.$ ALCL), which responds well to the cyclophosphamide, doxorubicin, vincristine, and prednisone (CHOP) regimen [9]. Among the various entities of PTCLs, adult T-cell leukemialymphoma (ATL) harbors the worst prognosis [10]. Here, we will discuss novel agents that have been developed for the treatment of ATL and other PTCLs, mainly focusing on mogamulizumab/KW-0761, which is a humanized monoclonal antibody targeting $\mathrm{CC}$ chemokine receptor 4 (CCR4) that has been actively developed for clinical use in Japan and the United States.

\section{PTCL and Novel Agents}

PTCL-not otherwise specified (PTCL-NOS) and angioimmunoblastic T-cell lymphoma (AITL), which are the most common subtypes of PTCL (PTCL-NOS, $26 \%$; AITL, $19 \%$ ), show a poor prognosis with 5-year overall survival (OS) and failure-free survival (FFS) of about $30 \%$ and $20 \%$, respectively [10]. Several new agents have recently been developed for the treatment of PTCL, mainly in patients with relapsed or refractory disease. Such agents have various mechanisms of action, including an immunomodulator (lenalidomide), a proteasome inhibitor (bortezomib), histone deacetylase inhibitors (vorinostat, romidepsin, panobinostat), antifolate (pralatrexate), and biologics including antibodies and antibody-toxin/drug conjugates (alemtuzumab, siplizumab, denileukin diftitox, and brentuximab vedotin) as well as nucleoside analogs such as fludarabine, gemcitabine, nelarabine, and forodesine [11]. Of these agents, pralatrexate and romidepsin have been recently approved by the U.S. Food and Drug Administration (FDA) and are now being used in the U.S. for the treatment of relapsed or refractory PTCL. In 2011, brentuximab vedotin (formerly known as SGN-35) was also approved for the treatment of relapsed or refractory ALCL and Hodgkin lymphoma.

ATL has the worst prognosis among PTCL, with 5-year OS and FFS of $14 \%$ and $12 \%$, respectively [10]. ATL is a peripheral T-cell malignancy associated with human T-cell lymphotropic virus type I (HTLV-1), and is relatively frequent in southwestern Japan, West Africa, the Caribbean islands, and Brazil, which are HTLV-1 endemic areas [12]. It is estimated that there are about 1.2 million HTLV-1 carriers in Japan, of whom a few percent develop ATL [13], and approximately 700 to 1000 people die of this disease per year [14]. ATL is classified into four disease subtypes (acute, lymphoma, chronic, and smoldering), based on clinical features including leukemic changes, high lactate dehydrogenase, hypercalcemia and organ infiltration, and the median survival time varies according to the disease type: acute type, 6 months; lymphoma type, 10 months; chronic type, 24 months; and smoldering type, 3 years or more [15]. It is recommended that treatment strategies should be selected according to the disease subtype [15]. In Japan, the acute type, lymphoma type, and chronic type with unfavorable prognostic factors have been regarded as aggressive ATL subtypes requiring immediate treatment, and intensive combination chemotherapy or allogeneic hematopoietic stem-cell transplantation are generally recommended therapeutic options [16].

The Japan Clinical Oncology Group-Lymphoma Study Group (JCOG-LSG) has been investigating the efficacy of combination chemotherapy for aggressive lymphomas including ATL or for ATL alone since the early 1980s. At the start of the investigation, CHOP-like regimens were evaluated because ATL was considered to be a type of NHL, but the outcome was poor [17]. Then, the LSG15 regimen consisting of the drugs used in the CHOP regimen plus four other drugs (ranimustine, vindesine, etoposide, and carboplatin) with the prophylactic use of granulocyte colonystimulating factor (G-CSF) was evaluated. In a phase III trial, JCOG9801, this dose-intensified multiagent chemotherapy regimen was shown to be more effective than CHOP-14 regimen, with a complete response rate of $40 \%$, 3 -year OS of $24 \%$, and median survival time of 12.7 months $[18,19]$. However, since the outcome of this dose-intensified regimen was still inferior to that in other PTCLs and B-cell lymphomas, further improvement is necessary. In Western countries, combination therapy with interferon- $\alpha$ and zidovudine has been widely used for all disease subtypes of ATL. A recently published meta-analysis suggested the effectiveness of this combination therapy for ATL, especially leukemic forms such as acute and chronic types [20].

Several new antibodies are currently under development for the treatment of T-cell lymphoma. They are based on the unique immunophenotypic features of ATL cells, which express mature T-cell antigens such as CD2, CD25 (interleukin [IL]-2 receptor), and CD52. Because of the unique intense expression of CD25 compared to that in other PTCL, monoclonal antibodies targeting the IL-2 receptor (anti-Tac), either radiolabeled or unlabelled (daclizumab), have been tested in patients with relapsed or refractory ATL. However, the clinical efficacy appears to be limited [21]. An anti-CD2 monoclonal antibody (siplizumab) [22], anti-CD52 antibody (alemtuzumab) [23, 24], and anti-transferrin receptor antibody (A24) [25] are also under development, but data are currently limited.

\section{Currently Available Therapeutic Agents for ATL}

Pentostatin and sobuzoxane are chemotherapeutic agents that were previously approved for the treatment of ATL in Japan. Pentostatin, a purine nucleoside analog that inhibits adenosine deaminase, has been reported to be effective for 
T-cell malignancies, including T-cell prolymphocytic leukemia, CTCL, and PTCL [26]. The clinical efficacy of pentostatin was evaluated in patients with ATL from the 1980s to 1990 s, and a phase II study of pentostatin revealed a response rate of $32 \%$ (10 of 31) in patients with relapsed or refractory ATL [27]. Other drugs that are often used in patients with relapsed or refractory ATL are some combination chemotherapy regimens, including EPOCH (etoposide, prednisolone, vincristine, cyclophosphamide, and doxorubicin) and ESHAP (etoposide, methylprednisolone, high-dose cytarabine and cisplatin); however, there is no apparent evidence of an advantage of these combination chemotherapies over other therapeutic options. In the U.S., pralatrexate and romidepsin have been approved for the treatment of PTCL and can also be used for ATL. The efficacy of these drugs for ATL is not clear because they have been evaluated only in a very limited number of patients (the efficacy of pralatrexate was evaluated in a clinical study in one patient) [28].

\section{CCR4 as a Novel Therapeutic Target}

Chemokines act as signaling molecules in the migration and tissue homing of various leukocytes. Among them, thymus and activation-regulated chemokine (TARC) and monocytederived chemokine (MDC) induce the selective recruitment of distinct subsets of T-cells through triggering of a chemokine receptor, CCR4. CCR4 is a seven-transmembrane Gprotein coupled receptor and selectively expressed on Th2 cells and regulatory $\mathrm{T}$ cells [29, 30]. The expression on normal cells such as Th2 cells can be partly regulated by the ligand, especially MDC [31], while the regulation by the ligands on cancer cells are not yet understood. Ishida et al. analyzed 103 patients with ATL, and found that tumor cells from about $90 \%$ of patients showed CCR4 expression [32]. They also found that patients with CCR4-positive ATL were more likely to have skin infiltration and had a worse outcome than those with CCR4-negative ATL, indicating that CCR4 played an important pathogenetic role in ATL [32]. In addition, Yoshie et al. found that the expression of CCR4 was increased in association of HTLV-1 and showed a relationship to Fra-2/Jun D which induces downstream genes such as c-Myb and SOX4, and MDM2 which promotes growth and inhibits apoptosis [33]. CCR4 is also expressed on other types of PTCL (29\% of total cases; PTCL-NOS, 38 \%; AITL, 35 \%; ALK ${ }^{-}$ALCL, $67 \%$; mycosis fungoides [MF], $41 \%$ ) [34]. Jones et al. independently reported that some types of PTCL expressed CCR4, as well [35]. In addition, analysis of 50 patients with PTCL-NOS revealed that CCR4-positive patients had significantly shorter survival than CCR4-negative patients [34]. Nakagawa et al. analyzed 51 patients with PTCL-NOS using the array comparative genomic hybridization technique, and found that patients with PTCL-NOS with genomic aberrations had a significantly higher frequency of CCR 4 positivity and a worse outcome than those with PTCL-NOS without genomic aberrations [36]. These findings resemble those observed in patients with ATL. Although the role of CCR4 in the tumorigenesis and progression of PTCL-NOS has not been fully elucidated, CCR4 seems to be a promising target molecule in the treatment of PTCL as well as in ATL.

\section{Clinical Trials of Mogamulizumab}

Mogamulizumab/KW-0761 is a humanized monoclonal antibody that recognizes the $\mathrm{N}$-terminal region of human CCR4 [37-39]. It is a therapeutic antibody produced using a novel glycoengineering technology that enhances antibodydependent cellular cytotoxic (ADCC) activity [40]. Mogamulizumab and its human-mouse chimeric version, KM2760, showed potent antitumor activity mediated by enhanced ADCC against ATL cell lines and primary ATL cells in vitro and in vivo [39, 41, 42].

A phase I clinical study (0761-0501 Study: ClinicalTrials.gov Identifier NCT00355472) has been conducted in patients with CCR4-positive relapsed PTCL, including ATL [43•]. The primary objectives of the study were to assess the safety of mogamulizumab, and analyze its maximum tolerated dose (MTD) and pharmacokinetics. The secondary objectives were to determine the best overall response rate (ORR) and progression-free survival (PFS). Mogamulizumab was intravenously administered once a week for 4 weeks at four dose levels $(0.01,0.1,0.5$, and $1.0 \mathrm{mg} / \mathrm{kg}$ ) according to the conventional $3+3$ design. Enrolled in the study were 16 patients, of whom 13 had ATL (11 acute type, 2 lymphoma type), 1 had tumor-stage MF, and 2 had PTCLNOS. All 16 patients receiving mogamulizumab were included in the safety and efficacy analyses. No dose-limiting toxicity (DLT) was observed in any of the 13 patients who received mogamulizumab at a dose of $0.01-1.0 \mathrm{mg} / \mathrm{kg}$, and thus MTD was not reached. Then, three additional patients were enrolled to receive $1.0 \mathrm{mg} / \mathrm{kg}$, the highest dose. One patient showed grade 4 neutropenia, grade 3 febrile neutropenia, and grade 3 skin eruption, but these adverse events occurred in only 1 of the 6 patients who received a dose of $1.0 \mathrm{mg} / \mathrm{kg}$, indicating that this drug would be tolerated at least up to $1.0 \mathrm{mg} / \mathrm{kg}$. The best ORR in the total 16 patients was $31 \%$ (of those, 2 had complete response [CR] and 3 had partial response [PR]), and the best ORR was also $31 \%$ in patients with ATL (of those, 2 had CR and another 2 had PR). Pharmacokinetic analysis revealed a plasma mogamulizumab trough concentration of $7.5-19.6 \mu \mathrm{g} / \mathrm{mL}$ after the 1 st to 4 th administration of mogamulizumab at a dose of $1.0 \mathrm{mg} / \mathrm{kg}$. These concentrations were sufficient to kill 
primary ATL cells by ADCC activity in vitro $(10 \mu \mathrm{g} / \mathrm{mL})$. After the 4th administration of mogamulizumab at a dose of $1.0 \mathrm{mg} / \mathrm{kg}$, its plasma half-life was approximately 18 days, which is comparable to the half-life (14 to 21 days) of endogenous human IgG. Lastly, although MTD was not reached, a tendency toward an increased incidence of grade 3 or higher toxicity was observed at $1.0 \mathrm{mg} / \mathrm{kg}$. Therefore, it was concluded that a dose of $1.0 \mathrm{mg} / \mathrm{kg}$ should be recommended for a subsequent phase II trial of this novel agent [44].

A subsequent phase II study of mogamulizumab (0761002 Study: ClinicalTrials.gov Identifier NCT 00920790) was conducted in patients with CCR4-positive relapsed ATL [45 $]$. The primary endpoint was the best ORR, and the secondary endpoints included the best response of each disease site such as peripheral blood ATL cells, skin and nodal/extranodal lesions as well as PFS and OS. It was planned for 25 patients to be enrolled for efficacy analysis, assuming the expected ORR of $30 \%$ with a $5 \%$ threshold response rate. Mogamulizumab was intravenously administered once a week for 8 weeks at a dose of $1.0 \mathrm{mg} / \mathrm{kg}$. In this study, 28 patients in total were enrolled. Of these, 27 patients who received mogamulizumab were included in the safety analysis, and 26 patients, excluding 1 patient who was judged ineligible for enrollment after starting mogamulizumab administration, were evaluated in the efficacy analysis. Of the 27 patients who received mogamulizumab, 14 had acute type, 6 lymphoma type, and 7 chronic type with unfavorable prognostic factors. The best ORR was $50 \%(13 / 26)$ including $8 \mathrm{CR}$. With the lower limit of the $95 \%$ confidence interval ( $30 \%$ to $70 \%$ ) exceeding the threshold response rate of $5 \%$, the clinical efficacy of mogamulizumab was confirmed. Responses according to disease sites were $100 \%$ (of 13 patients, all CR) for peripheral blood, $63 \%$ (of 8 patients, $3 \mathrm{CR}$ and $2 \mathrm{PR}$ ) for skin, and $25 \%$ (of 12 patients, $3 \mathrm{CR} / \mathrm{CRu}$ ) for nodal and extranodal lesions. Median PFS and OS were 5.2 and 13.7 months, respectively. The best ORR was also calculated for each disease subtype, giving $43 \%$ in patients with acute type (of 14 patients, $5 \mathrm{CR}$ and $1 \mathrm{PR}$ ), $33 \%$ in patients with lymphoma type (of 6 patients, $1 \mathrm{CR}$ and $1 \mathrm{PR}$ ), and $83 \%$ in patients with unfavorable chronic type (of 6 patients, 2 $\mathrm{CR}$ and $3 \mathrm{PR}$ ). Thus, it was demonstrated that mogamulizumab induced favorable responses in patients with any disease subtype of ATL. In addition, for each age group, the best ORR was $39 \%$ (of 13 patients, $3 \mathrm{CR}$ and $2 \mathrm{PR}$ ) in patients younger than 65 years, and $62 \%$ (of 13 patients, 5 $\mathrm{CR}$ and $3 \mathrm{PR}$ ) in patients 65 years or older. The most common adverse events observed during the study were lymphopenia (96\%), neutropenia (52\%), and thrombocytopenia $(52 \%)$ as hematologic toxicity, and acute infusion reaction $(89 \%)$ and skin eruption $(63 \%)$ as nonhematologic toxicity. There was no death related to mogamulizumab in either the phase I or phase II study. Of 8 serious adverse events with a relationship to mogamulizumab in the phase I and II studies, 5 events including 4 cases of skin eruption and 1 case of Stevens-Johnson syndrome occurred during the phase II study. However, these reactions were manageable with supportive measures including corticosteroid or other drugs in all patients. Considering the seriousness of the disease, even skin eruption might be considered acceptable for the treatment of ATL by treating physicians, while close and appropriate follow-up of the event is necessary. Elucidation of the mechanism of skin eruption and preventive measures against it are awaited.

In the U.S., a phase I/II study (ClinicalTrials.gov Identifier: NCT00888927) in patients with relapsed or refractory PTCL including CTCL has been conducted [46]. Mogamulizumab was well tolerated at doses of $0.1-1.0 \mathrm{mg} / \mathrm{kg}$ in 42 patients including 1 with PTCL-NOS. MTD was not reached and thus $1.0 \mathrm{mg} / \mathrm{kg}$ was chosen for subsequent studies. A promising ORR of $42 \%$ (of 38 evaluable patients with CTCL, 3 CR and 13 PR) was achieved, although expression of CCR4 on lymphoma cells was not mandatory for patient enrolment in this particular phase I/II study. Regarding subtypes of CTCL, ORR in Sezary syndrome (SS) patients was $50 \%$ and in MF patients was $36 \%$. Eighty-seven percent of SS patients had a response in peripheral blood, with $50 \% \mathrm{CR}$. Further study of mogamulizumab is warranted in patients with nodal PTCL as well as CTCL.

\section{Conclusions}

It is evident that there are limitations to improvement of the treatment outcome of PTCL, especially ATL, with the currently available chemotherapeutic agents alone. Mogamulizumab has a less severe toxicity profile and induces a high response rate in patients with ATL, even in elderly patients.
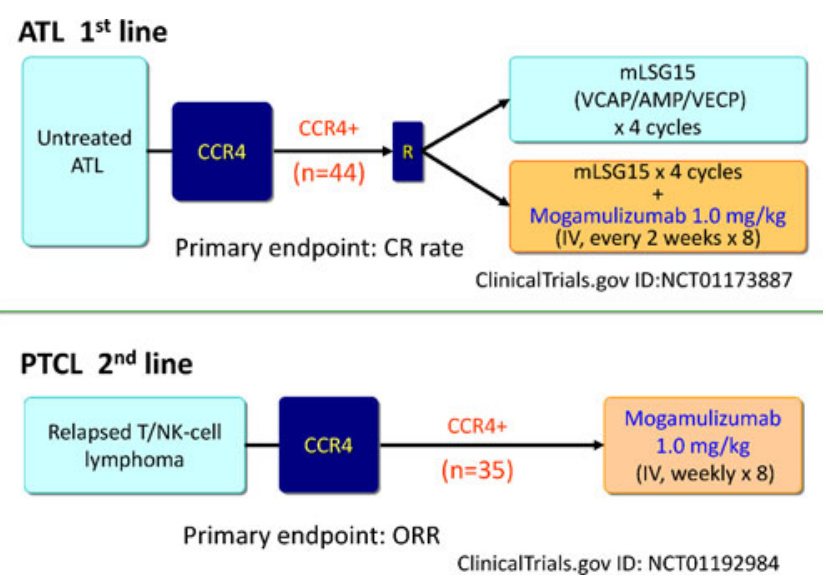

Fig. 1 Ongoing clinical studies of mogamulizumab/KW-0761 for peripheral T-cell lymphomas in Japan 
Therefore, it may provide an effective treatment option for the disease, especially for elderly patients who are not eligible for intensive chemotherapy or hematopoietic stem-cell transplantation.

In Japan, based on the results of the clinical studies mentioned above, a single-arm phase II study of mogamulizumab monotherapy in patients with CTCL and PTCL (ClinicalTrials.gov Identifier: NCT01192984) and a randomized phase II study of dose-intensified combination chemotherapy (mLSG15 regimen) with or without mogamulizumab in untreated patients with ATL (ClinicalTrials.gov Identifier: NCT01173887) are being conducted as shown in Fig. 1. Patient enrollment has already been completed in these phase II studies. In the U.S., based on the aforementioned phase I/II study in patients with relapsed or refractory CTCL, a pivotal phase III study in patients with relapsed or refractory CTCL is being planned. In conclusion, mogamulizumab is expected to provide new, promising treatment options in patients with ATL and other T-cell lymphomas.

Acknowledgments We thank all the investigators who participated in the multicenter clinical trials of mogamulizumab/KW-0761 in Japan. Clinical studies of mogamulizumab were sponsored by Kyowa Hakko Kirin Co. Ltd., Tokyo, Japan.

Disclosure K. Tobinai: research grant from Kyowa Hakko Kirin Co. Ltd. and board membership for Merck, Mundipharma, Zenyaku, Genzyme, Eisai, Symbio, Eli Lilly, Celgene, Kyowa-Kirin, Biomedics, and Solasia Pharma; T. Takahashi: employee of Kyowa Hakko Kirin Co. Ltd.; S. Akinaga: employee of Kyowa Hakko Kirin Co. Ltd.

Open Access This article is distributed under the terms of the Creative Commons Attribution License which permits any use, distribution, and reproduction in any medium, provided the original author(s) and the source are credited.

\section{References}

Papers of particular interest, published recently, have been highlighted as:

- Of importance

1. Ascani S, Zinzani PL, Gherlinzoni F, et al. Peripheral T-cell lymphomas. Clinico-pathologic study of 168 cases diagnosed according to the R.E.A.L. Classification. Ann Onco. 1997;8:583-92.

2. Anderson JR, Armitage JO, Weisenburger DD. Epidemiology of the non-Hodgkin's lymphomas: distributions of the major subtypes differ by geographic locations. Non-Hodgkin's Lymphoma Classification Project. Ann Oncol. 1998;9:717-20.

3. Nakamura S, Koshikawa T, Koike K, et al. Phenotypic analysis of peripheral T cell lymphoma among the Japanese. Acta Pathol Jpn. 1993;43:396-412.

4. Aoki R, Karube K, Sugita Y, et al. Distribution of malignant lymphoma in Japan: analysis of 2260 cases, 2001-2006. Pathol Int. 2008;58:174-82.

5. Swerdlow SH, Campo E, Harris NL, et al. WHO classification of tumors of haematopoietic and lymphoid tissues. Lyon, France: IARC Press; 2008.
6. National Comprehensive Cancer Network (NCCN). Non-Hodgkin's lymphomas. NCCN clinical practice guidelines in oncology. v.1.2010. Fort Washington, PA: NCCN; 2010.

7. Coiffier B, Lepage E, Brière J, et al. CHOP chemotherapy plus rituximab compared with $\mathrm{CHOP}$ alone in elderly patients with diffuse large B-cell lymphoma. N Engl J Med. 2002;346:235-42.

8. Friedberg JW, Cohen P, Chen L, et al. Bendamustine in patients with rituximab-refractory indolent and transformed non-Hodgkin's lymphoma: results from a phase II multicenter, single-agent study. J Clin Oncol. 2008;26:204-10.

9. Sandlund JT, Pui CH, Santana VM, et al. Clinical features and treatment outcome for children with $\mathrm{CD} 30+$ large-cell nonHodgkin's lymphoma. J Clin Oncol. 1994;12:895-8.

10. Vose J, Armitage J, Weisenburger D. International T-Cell Lymphoma Project. International peripheral T-cell and natural killer/T-cell lymphoma study: pathology findings and clinical outcomes. J Clin Oncol. 2008;26:4124-30.

11. Foss FM, Zinzani PL, Vose JM, et al. Peripheral T-cell lymphoma. Blood. 2011;117:6756-67.

12. Sonoda S, Li HC, Tajima K. Ethnoepidemiology of HTLV-1 related diseases: ethnic determinants of HTLV-1 susceptibility and its worldwide dispersal. Cancer Sci. 2011;102:295-301.

13. Iwanaga $M$, Watanabe $T$, Utsunomiya $A$, et al. Human T-cell leukemia virus type I (HTLV-1) proviral load and disease progression in asymptomatic HTLV-1 carriers: a nationwide prospective study in Japan. Blood. 2010;116:1211-9.

14. Yamaguchi K, Watanabe T. Human T lymphotropic virus type-I and adult T-cell leukemia in Japan. Int J Hematol. 2002;76 Suppl 2:240-5.

15. Shimoyama M. Diagnostic criteria and classification of clinical subtypes of adult T-cell leukaemia-lymphoma: A report from the Lymphoma Study Group (1984-87). Br J Haematol. 1991;79:428-37.

16. Tsukasaki K, Hermine O, Bazarbachi A, et al. Definition, prognostic factors, treatment, and response criteria of adult T-cell leukemia-lymphoma: a proposal from an international consensus meeting. J Clin Oncol. 2009;27:453-9.

17. Tobinai K. Clinical trials for human T-cell lymphotropic virus type I-associated peripheral T-cell lymphoma in Japan. Semin Hematol. 2010;47 Suppl 1:S5-7.

18. Yamada Y, Tomonaga M, Fukuda H, et al. A new G-CSFsupported combination chemotherapy, LSG15, for adult T-cell leukaemia-lymphoma: Japan Clinical Oncology Group Study 9303. Br J Haematol. 2001;113:375-82.

19. Tsukasaki K, Utsunomiya A, Fukuda H, et al. VCAP-AMP-VECP compared with biweekly CHOP for adult T-cell leukemialymphoma: Japan Clinical Oncology Group Study JCOG9801. J Clin Oncol. 2007;25:5458-64.

20. Bazarbachi A, Plumelle Y, Carlos Ramos J, et al. Meta-analysis on the use of zidovudine and interferon-alfa in adult T-cell leukemia/ lymphoma showing improved survival in the leukemic subtypes. $\mathrm{J}$ Clin Oncol. 2010;28:4177-83.

21. Waldmann TA. Daclizumab (anti-Tac, Zenapax) in the treatment of leukemia/lymphoma. Oncogene. 2007;26:3699-703.

22. Zhang Z, Zhang M, Ravetch JV, et al. Effective therapy for a murine model of adult T-cell leukemia with the humanized antiCD2 monoclonal antibody, MEDI-507. Blood. 2003;102:284-8.

23. Zhang Z, Zhang M, Goldman CK, et al. Effective therapy for a murine model of adult T-cell leukemia with the humanized anti-CD52 monoclonal antibody, Campath-1H. Cancer Res. 2003;63:6453-7.

24. Ravandi F, Faderl S. Complete response in a patient with adult T-cell leukemia (ATL) treated with combination of alemtuzumab and pentostatin. Leuk Res. 2006;30:103-5.

25. Callens C, Moura IC, Lepelletier Y, et al. Recent advances in adult T-cell leukemia therapy: focus on a new anti-transferrin receptor monoclonal antibody. Leukemia. 2008;22:42-8.

26. Dearden CE. Role of single-agent purine analogues in therapy of peripheral T-cell lymphomas. Semin Hematol. 2006;43:S22-6. 
27. Tobinai K. Current management of adult T-cell leukemia/lymphoma. Oncology (Williston Park). 2009;23:1250-6.

28. O'Connor OA, Pro B, Pinter-Brown L, et al. Pralatrexate in patients with relapsed or refractory peripheral T-cell lymphoma: results from the pivotal PROPEL study. J Clin Oncol. 2011;29:1182-9.

29. D'Ambrosio D, Iellem A, Bonecchi R, et al. Selective up-regulation of chemokine receptors CCR4 and CCR8 upon activation of polarized human type 2 Th cells. J Immunol. 1998;161:5111-5.

30. Iellem A, Mariani M, Lang R, et al. Unique chemotactic response profile and specific expression of chemokine receptors CCR4 and CCR 8 by CD4(+)CD25(+) regulatory T cells. J Exp Med. 2001;194:847-53.

31. Mariani M, Lang R, Binda E, et al. Dominance of CCL22 over CCL17 in induction of chemokine receptor CCR4 desensitization and internalization on human Th2 cells. Eur J Immunol. 2004;34:231-40.

32. Ishida $\mathrm{T}$, Utsunomiya $\mathrm{A}$, Iida $\mathrm{S}$, et al. Clinical significance of CCR4 expression in adult T-cell leukemia/lymphoma: its close association with skin involvement and unfavorable outcome. Clin Cancer Res. 2003;9:3625-34.

33. Nakayama T, Hieshima K, Arao T, et al. Aberrant expression of Fra-2 promotes CCR4 expression and cell proliferation in adult T-cell leukemia. Oncogene. 2008;27:3221-32.

34. Ishida $\mathrm{T}$, Inagaki $\mathrm{H}$, Utsunomiya A, et al. CXC chemokine receptor 3 and CC chemokine receptor 4 expression in T-cell and NK-cell lymphomas with special reference to clinicopathological significance for peripheral T-cell lymphoma, unspecified. Clin Cancer Res. 2004;10:5494-500.

35. Jones D, O'Hara C, Kraus MD, et al. Expression pattern of T-cellassociated chemokine receptors and their chemokines correlates with specific subtypes of T-cell non-Hodgkin lymphoma. Blood. 2000;96:685-90.

36. Nakagawa M, Nakagawa-Oshiro A, Karnan S, et al. Array comparative genomic hybridization analysis of PTCL-U reveals a distinct subgroup with genetic alterations similar to lymphoma-type adult Tcell leukemia/lymphoma. Clin Cancer Res. 2009;15:30-8.

37. Imai T, Nagira M, Takagi S, et al. Selective recruitment of CCR4bearing Th2 cells toward antigen-presenting cells by the CC chemokines thymus and activation-regulated chemokine and macrophage-derived chemokine. Int Immunol. 1999;11:81-8.

38. Niwa R, Shoji-Hosaka E, Sakurada M, et al. Defucosylated chimeric anti-CC chemokine receptor $4 \mathrm{IgG} 1$ with enhanced antibody-dependent cellular cytotoxicity shows potent therapeutic activity to T-cell leukemia and lymphoma. Cancer Res. 2004;64:2127-33.

39. Ishii $T$, Ishida $T$, Utsunomiya $A$, et al. Defucosylated humanized anti-CCR4 monoclonal antibody KW-0761 as a novel immunotherapeutic agent for adult T-cell leukemia/lymphoma. Clin Cancer Res. 2010;16:1520-31.

40. Shinkawa T, Nakamura K, Yamane N, et al. The absence of fucose but not the presence of galactose or bisecting $\mathrm{N}$-acetylglucosamine of human IgG1 complex-type oligosaccharides shows the critical role of enhancing antibody-dependent cellular cytotoxicity. J Biol Chem. 2003;278:3466-73.

41. Yano H, Ishida T, Imada K, et al. Augmentation of antitumour activity of defucosylated chimeric anti-CCR4 monoclonal antibody in SCID mouse model of adult T-cell leukaemia/lymphoma using G-CSF. Br J Haematol. 2008;140:586-9.

42. Ito A, Ishida T, Utsunomiya A, et al. Defucosylated anti-CCR4 monoclonal antibody exerts potent ADCC against primary ATLL cells mediated by autologous human immune cells in NOD/Shi-scid, IL-2R gamma(null) mice in vivo. J Immunol. 2009;183:4782-91.

43. - Yamamoto K, Utsunomiya A, Tobinai K, et al. Phase I study of KW-0761, a defucosylated humanized anti-CCR4 antibody, in relapsed patients with adult T-cell leukemia-lymphoma and peripheral T-cell lymphoma. J Clin Oncol. 2010;28:1591-8. This manuscript describes the results of a phase I study of mogamulizumab (KW-0761) in ATL and PTCL, showing encouraging therapeutic efficacy with acceptable toxicity profiles.

44. Yamamoto K, Tobinai K, Akinaga S, et al. Reply to R. Suzuki. J Clin Oncol. 2010;29:8356.

45. • Ishida T, Joh T, Uike N, et al. Defucosylated anti-CCR4 monoclonal antibody (KW-0761) for relapsed adult T-cell leukemia-lymphoma: a multicenter phase II study. J Clin Oncol. 2012;30:837-42. This manuscript describes the results of a phase II study of mogamulizumab/KW-0761 in relapsed patients with ATL, yielding a best overall response rate of $50 \%$ and median progression-free survival of 5.2 months.

46. Duvic M, Pinter-Brown L, Foss FM, et al.: Results of a phase $1 / 2$ study for KW-0761, a monoclonal antibody directed against CC chemokine receptor type 4 (CCR4), in CTCL patients. [abstract 962]. Presented at the 52nd Annual Meeting of the American Society of Hematology. Orlando, USA; December 4-7, 2010 . 